\title{
Anticancer Gene-engineered MSC-mediated Cancer Cell Death: An Imaging Demonstration
}

\author{
Xu-Yong Sun ${ }^{1 *}$, Zhuang Chen ${ }^{2 *}$, Mani R. Moniri ${ }^{3}$, Hong Lu ${ }^{3}$, Long-Jun Dai ${ }^{3 \#}$, Garth L. Warnock ${ }^{3}$ \\ ${ }^{1}$ Institute of Transplant Medicine1, 303 Hospital of PLA, Nanning, Guangxi, China \\ ${ }^{2}$ Laboratory of Infection and Immunity, Affiliated Hospital of Luzhou Medical College, Luzhou, Sichuan, China \\ ${ }^{3}$ Department of Surgery, University of British Columbia, Vancouver, Canada \\ Email: \#jdai@mail.ubc.ca \\ Received 2012
}

\begin{abstract}
This study was performed to demonstrate the transportation of an engineered MSC-produced intracellular anticancer gene product between mesenchymal stem cell (MSC) and cancer cells. MSC-mediated anticancer strategy has held great promise owing to MSCs' capacity of tumor-directed migration and the availability of specific anticancer genes. All anticancer genes that have been used in previous MSC-mediated anticancer studies were limited in functioning via extracellular mechanisms, mainly because of the restriction by cell membrane to macromolecules including proteins. In order to apply the majority of potent anticancer genes to the MSCmediated anticancer system, a specifically designed expression vector which bears an intracellular anticancer gene, PTEN, is utilized to demonstrate the feasibility of the system in cancer therapies. A transacting activator of transcription (TAT) was introduced into an expression vector followed by a segment for PTEN-RFP fusion protein. A direct demonstration of PTEN-RFP transportation between MSC and cancer cells was obtained from direct co-cultures. A marked cancer cell death was observed in indirect co-cultures with conditioned media from PTEN-transfected MSCs. The demonstration of PTEN-engineered MSC-produced PTEN transportation indicates the feasibility of applying intracellular anticancer gene expression system in MSC-mediated strategies for cancer therapy.
\end{abstract}

Keywords: Genetic Engineering; Mesenchymal Stem Cells; Gene Therapy; Cancer Therapy; PTEN.

\section{Introduction}

Cancer remains one of the leading causes of mortality and morbidity throughout the world. To a significant extent, current conventional cancer therapies are symptomatic and passive in nature. The major obstacle to the development of effective cancer therapy is believed to be the absence of sufficient tumor specificity. Since the discovery of tumor-oriented homing capacity of mesenchymal stem cells (MSCs), the application of specific anticancer gene-engineered MSCs has held great promise for cancer therapies [1]. MSC-mediated anticancer therapy relies on tumor-specific selectivity provided by MSCs and MSC-carried anticancer agents. Homed directly at the tumor microenvironment, engineered MSCs are able to express and/or release anticancer agents to constantly act on the adjacent tumor cells. Based on the mechanisms of tumor suppression, MSC-mediated anticancer agents are classified into following categories: (1) immunostimulation, such as CX3CL1 [2], IFN [3,4], IL2 [5], IL7 [6] and IL12 [7]. (2) prodrug conversion, such as CD [8] and HSV-tk [9]. And (3) apoptosis induction, such as IL18 [10], NK4 [11] and TRAIL [12-14]. All of these anticancer agents used in previous studies are produced by engineered MSCs and act on targeted tumor cells extracellularly. However, the majority of anticancer gene products act on tumor cells through intracellular mechanisms, such as P53, Myc and phosphatase and tensin homolog (PTEN). When MSCs are used as a vehicle to deliver intracellular anticancer

\footnotetext{
*These authors contributed equally to this work.
}

${ }^{\#}$ Corresponding author. genes, the anticancer gene products must be produced in MSCs and secreted into extracellular space and then penetrate into adjacent tumor cells passing through biological membrane, which is a natural barrier for most macromolecules including peptides and proteins. In this conference, we present a uniquely designed expression vector and use PTEN-engineered MSCs as an example to demonstrate cancer cell death induced by intracellular anticancer genes.

\section{Materials and Methods}

\subsection{Cells and Culture Conditions}

MSCs were isolated from human pancreas and ex vivo expanded as previously described [14]. Based on the minimal criteria for defining human MSCs established by the International Society of Cellular Therapy, these MSCs were verified by both membrane biomarker determination and functional differentiation. They fulfilled the characteristics of human MSCs, exhibiting positive expression of $\mathrm{CD} 44^{+}, \mathrm{CD}^{+} 3^{+}, \mathrm{CD}^{+} 5^{+}$, $\mathrm{CD}_{105^{+}}$and negative of CD34- The results of adipogenic and osteogenic differentiation also met the standards. MSCs were cultured in MEM with 10\% FCS, 2 mM L-glutamine and 1\% penicillin-streptomycin solution (all from Invitrogen, Carlsbad, CA, USA) and incubated at $37^{\circ} \mathrm{C}$ in a humidified, $5 \% \mathrm{CO}_{2}$ atmosphere.

A human pancreatic cancer cell line (Panc-1) was purchased from American Type Culture Collection (ATCC, Manassas, VA, USA) and used as target cells in the present study. Panc-1 cells were maintained as suggested by ATCC and their culture 
condition was kept consistent with the MSCs. In our previous study, these cells were verified negative expression for both death receptors (DR) 4 and 5, and showed no response to TNF-related apoptosis-inducing ligand (TRAIL) [15].

\subsection{Construction of PTEN-bearing Expression Vector}

Mammalian expression plasmid pDsRed1-N1 was used as the backbone structure. As illustrated in Figure 1, an 18 amino acid leading sequence and an 11 amino acid transacting activator of transcription (TAT) were inserted at the multiple cloning site followed by human PTEN (403 amino acids). A TAT-PTENRFP fusion protein was designated and the predicted molecular weight was around $74 \mathrm{kd}$.

\subsection{Plasmid Transfection of MSCs and Determination of Cell Viability}

PTEN-bearing expression plasmid was transfected into MSCs with TransIT-2020 (Mirus, Madison, WI, USA) as suggested by the manufacturer. Briefly, the MSCs were plated at $6 \times 10^{5}$ per well in six-well plates in $3 \mathrm{ml}$ of MEM medium and dated as day 0 . On day 1 , the cells were transfected with $2.5 \mu \mathrm{g}$ of PTEN-RFP or the same plasmid without PTEN as controls. MSCs transfected with PTEN-RFP plasmid were named as MSC $^{P T E N}$. For the direct co-culture experiments, MSC $^{P T E N}$ were harvested with $0.25 \%$ trypsin-EDTA (Invitrogen) on day 2 . For the purpose of indirect co-culture and the assessment of PTEN expression, conditioned media and MSC $^{P T E N}$ were collected on day 3.

The cell viability was detected using a LIVE/DEAD Viability/Cytotoxicity Assay Kit (Invitrogen) as per the manufacturer's instruction with a slight modification. Briefly, a total of $1 \mathrm{x}$ $10^{5}$ Panc-1 cells were plated onto 24-well plates in $500 \mu \mathrm{l}$ of MEM medium on day 0 . For indirect co-cultures the media were replaced with $100 \%$ conditioned media (from both MSC $^{\text {PTEN }}$ and control MSCs) on day 1 . The cell viability assessments were performed on day 4 . The cultures were washed with PBS and then, freshly prepared working solution $(250 \mu \mathrm{l}$ per well containing $1 \mu \mathrm{M}$ calcein AM and $2 \mu \mathrm{M}$ EthD-1) was added directly to the cultures and incubated at room temperature for $10 \mathrm{~min}$ in the dark. The images were taken using a fluorescence microscope (IX71; Olympus, Markham, Ontario, Canada).

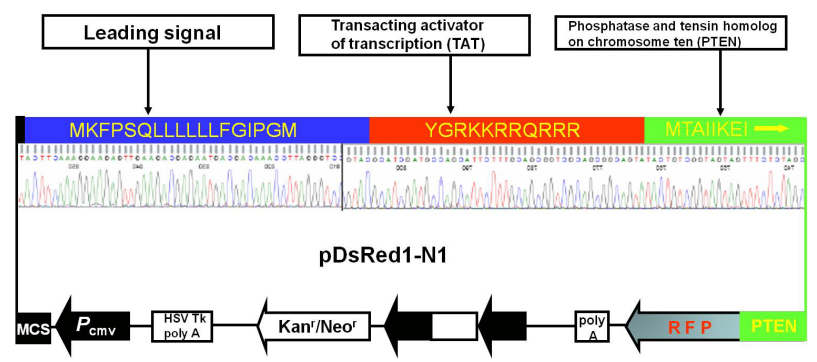

Figure 1. Structure of PTEN expression vector Plasmid pDsRed1N1 was used as the backbone structure. A leading signal (18 amino acids) was cloned into the multiple cloning site (MCS) followed by transacting activator of transcription (TAT, 11 amino acids). PTEN (403 amino acids) was inserted before the red fluorescence protein (RFP).

\section{Results}

\subsection{Identification of PTEN-bearing Vector}

The construction of PTEN-RFP expression vector was verified by DNA sequencing, and its functional expression in MSC ${ }^{P T E N}$ was confirmed by microscopic observation (Figure 2). Immunoblotting analysis of PTEN in MSC cell lysates showed two PTEN antibody reactive bands with molecular weights $47 \mathrm{kd}$ and 74kd respectively, indicating the endogenous PTEN expression and additional expression induced by PTEN-RFP transfection in MSCs.

\subsection{Demonstration of PTEN Travelling}

PTEN is an intracellular protein and all of its tumor suppressor effects take place intracellularly. It is essential to confirm that MSC $^{\text {PTEN }}$-produced PTEN is able to be secreted into the extracellular space and subsequently penetrate into adjacent cancer cells. To simulate the natural situation, cancer cells (Panc-1) were pre-labeled with carboxyfluorescein succinimidyl ester (CFSE) and seeded on culture plates one day earlier than the application of MSC ${ }^{P T E N}$ (Figure 2). Figure 3 demonstrates the PTEN travelling between Panc-1 and MSC ${ }^{\text {PTEN }}$.

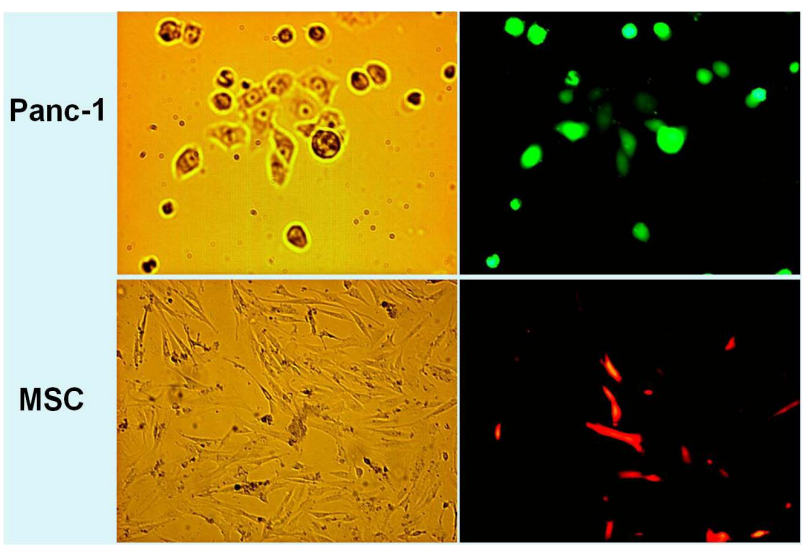

Figure 2. Imaging presentation of Panc-1 and MSCs prior to coculture Left and right panels show brightfield and fluorescence images respectively. Panc-1 cells were pre-labeled with CFSE (green) and MSCs were transfected with PTEN-RFP (red) expression vectors. (Original magnification, $x$ 400)

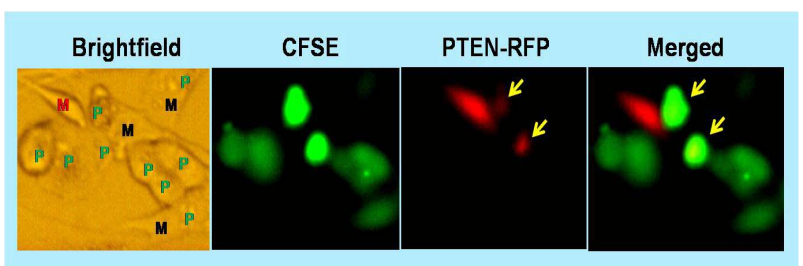

Figure 3. Demonstration of PTEN-RFP transportation between MSC and Panc-1 cells CFSE-lebeled Panc-1 cells were plated on day 1 and PTEN-RFP-transfected MSCs were seeded into the same plate on day 2. The images were taken on day 3 from the same observation field with different light sources. M: MSCs transfected with PTEN-RFP (red fluorescence protein); M: MSCs transfected with PTEN-RFP but not yet expressed; P: pancreatic cancer cells (Panc-1) pre-labeled with CFSE (green). The yellow arrows point two cancer cells which contain PTEN-RFP. 


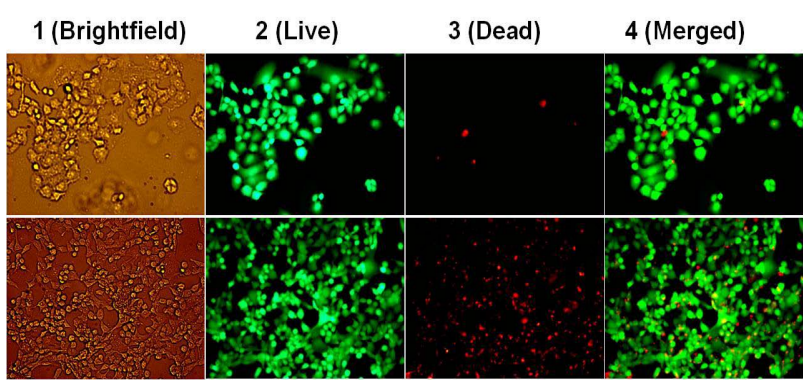

Figure 4. Panc-1 cell viability in indirect co-cultures with conditioned media Panc-1 cells were plated on day 1 . Conditioned media from native MSCs (top panel) and MSC ${ }^{P T E N}$ (bottom panel) were replaced on day 2 . The cell viability assessment was performed with LIVE/DEAD assay on day 4. Column 1 (brightfield): whole population of cells which were still attached to the culture surface during the assessment; column 2: live cells stained with calcein are green; column 3: dead cells stained with EthD-1 show red; column 4: merged images. (Original magnification, $x 400$ ).

\subsection{MSCPTEN-mediated Panc-1 cell Death in Indirect Co-cultures}

MSC $^{\text {PTEN }}$-mediated Panc-1 cell death was assessed in indirect co-cultures using conditioned media from control MSCs and MSC $^{\text {PTEN }}$. As shown in Figure 4, marked cell death was observed when Panc-1 cell were co-cultured with conditioned media from MSC $^{\text {PTEN }}$. It is worth noting that LIVE/DEAD assay only applies to the cells which remain on the culture surface during the staining. The detached cells, most of which are dead cells, are not included in the assessment.

\section{Discussion and Conclusion}

The tumor suppressor phosphatase and tensin homolog (PTEN) was discovered in 1997 [16,17]. The PTEN gene is located at chromosome 10q23.31. Loss of heterozygosity at 10q23 occurs frequently in many advanced-stage sporadic tumors; for example, approximately $70 \%$ in glioblastomas and $60 \%$ in advanced prostate cancers [17]. PTEN is also known as mutated in multiple advanced cancer 1 (MMAC1). It functions as the central negative regulator of PI3K-AKT-mTOR pathway in controlling apoptosis. PI3K-AKT-mTOR signaling pathway is the most frequently activated pathway in human cancers, because it promotes cell growth and contributes to the evasion of apoptosis, loss of cell cycle control and genomic instability during tumorigenesis. PTEN dephosphorylates $\mathrm{PIP}_{3}$ to $\mathrm{PIP}_{2}$ thereby directly opposing the activity of PI3K. Compared to other classical tumor suppressor genes, PTEN is haploinsufficient because a single copy is unable to prevent cancer. Loss of its heterozygosity or partial inhibition of its expression/activity is sufficient to promote carcinogenesis [18]. Thus, restoring PTEN function in cancer cells would break down the PTEN mutation-dependent cancer cell growth, and this holds great promise for cancer therapy.

Wide-type PTEN can be introduced into cells by using viral vectors [19] or a non-viral vector-mediated delivery such as urocanic acid-modified chitosan-mediated PTEN delivery via aerosol [20]. However, engineering MSCs with PTEN could strongly potentiate the efficacy of targeted cancer therapy [21]. For the first time, a cell permeable recombinant wide-type
PTEN was transfected into MSCs via fusion PTEN with transactivator of transcription (TAT). As demonstrated in Figure 3, MSC-produced PTEN-RFP appears in the adjacent cancer cells. This observation provides a direct evidence of PTEN-RFP travelling across multiple membranes. PTEN-engineered MSCmediated cancer cell death was also confirmed in indirect cocultures with Panc- 1 cells which are insensitive to TRAIL (Figure 4). This concise demonstration reveals the application of intracellular anticancer gene-bearing expression system in MSC-mediated cancer therapy, which potentially broadens the spectrum of anticancer gene-related cancer therapy.

In conclusion, the demonstration of PTEN-engineered MSC-produced PTEN transportation indicates the feasibility of applying intracellular anticancer gene expression system in MSC-mediated anticancer treatment. PTEN-induced Panc-1 cell death also suggests possible synergistic interactions of multiple anticancer genes to highly heterogeneous carcinomas.

\section{Acknowledgements}

This work was supported by the Guangxi Ministry of Science and Technology (0993003A, 0719002-2-7) and the VGH \& UBC Hospital Foundation. The authors are gratiful to Crystal Robertson for her assistance in preparing the manuscript.

\section{REFERENCES}

[1] M. R. Loebinger and S. M. Janes, "Stem cells as vectors for antitumour therapy," Thorax, vol. 65, pp. 362-9, Apr 2010.

[2] H. Xin, et al., "Targeted delivery of CX3CL1 to multiple lung tumors by mesenchymal stem cells," Stem Cells, vol. 25, pp. 1618-26, Jul 2007.

[3] C. Ren, et al., "Therapeutic potential of mesenchymal stem cells producing interferon-alpha in a mouse melanoma lung metastasis model," Stem Cells, vol. 26, pp. 2332-8, Sep 2008.

[4] S. Kidd, et al., "Mesenchymal stromal cells alone or expressing interferon-beta suppress pancreatic tumors in vivo, an effect countered by anti-inflammatory treatment," Cytotherapy, vol. 12, pp. 615-25, Sep 2010.

[5] K. Nakamura, et al., "Antitumor effect of genetically engineered mesenchymal stem cells in a rat glioma model," Gene Ther, vol. 11, pp. 1155-64, Jul 2004.

[6] S. Gunnarsson, et al., "Intratumoral IL-7 delivery by mesenchymal stromal cells potentiates IFNgamma-transduced tumor cell immunotherapy of experimental glioma," J Neuroimmunol, vol. 218, pp. 140-4, Jan 252010.

[7] X. Chen, et al., "A tumor-selective biotherapy with prolonged impact on established metastases based on cytokine gene-engineered MSCs," Mol Ther, vol. 16, pp. 749-56, Apr 2008.

[8] I. T. Cavarretta, et al., "Adipose tissue-derived mesenchymal stem cells expressing prodrug-converting enzyme inhibit human prostate tumor growth," Mol Ther, vol. 18, pp. 223-31, Jan 2010.

[9] H. Miletic, et al., "Bystander killing of malignant glioma by bone marrow-derived tumor-infiltrating progenitor cells expressing a suicide gene," Mol Ther, vol. 15, pp. 1373-81, Jul 2007.

[10] G. Xu, et al., "Adenoviral-mediated interleukin-18 expression in mesenchymal stem cells effectively suppresses the growth of glioma in rats," Cell Biol Int, vol. 33, pp. 466-74, Apr 2009.

[11] M. Kanehira, et al., "Targeted delivery of NK4 to multiple lung tumors by bone marrow-derived mesenchymal stem cells," Can- 
cer Gene Ther, vol. 14, pp. 894-903, Nov 2007.

[12] L. G. Menon, et al., "Human bone marrow-derived mesenchymal stromal cells expressing S-TRAIL as a cellular delivery vehicle for human glioma therapy," Stem Cells, vol. 27, pp. 2320-30, Sep 2009.

[13] M. R. Loebinger, et al., "Mesenchymal stem cell delivery of TRAIL can eliminate metastatic cancer," Cancer Res, vol. 69, pp. 4134-42, May 152009.

[14] X. Y. Sun, et al., "MSC(TRAIL)-mediated HepG2 cell death in direct and indirect co-cultures," Anticancer Res, vol. 31, pp. 3705-12, Nov 2011.

[15] X. Y. Sun, et al., "Mesenchymal stem cell-mediated cancer therapy: A dual-targeted strategy of personalized medicine," World J Stem Cells, vol. 3, pp. 96-103, Nov 262011.

[16] P. A. Steck, et al., "Identification of a candidate tumour suppressor gene, MMAC1, at chromosome 10q23.3 that is mutated in multiple advanced cancers," Nat Genet, vol. 15, pp. 356-62, Apr 1997.

[17] J. Li, et al., "PTEN, a putative protein tyrosine phosphatase gene mutated in human brain, breast, and prostate cancer," Science, vol. 275, pp. 1943-7, Mar 281997.

[18] M. Peyrou, et al., "PTEN in liver diseases and cancer," World J Gastroenterol, vol. 16, pp. 4627-33, Oct 72010.

[19] G. Pappas, et al., "Adenoviral-mediated PTEN expression radiosensitizes non-small cell lung cancer cells by suppressing DNA repair capacity," Cancer Gene Ther, vol. 14, pp. 543-9, Jun 2007.

[20] H. Jin, et al., "Urocanic acid-modified chitosan-mediated PTEN delivery via aerosol suppressed lung tumorigenesis in K-ras(LA1) mice," Cancer Gene Ther, vol. 15, pp. 275-83, May 2008.

[21] L. J. Dai, et al., "Potential implications of mesenchymal stem cells in cancer therapy," Cancer Lett, vol. 305, pp. 8-20, Jun 1 2011. 